027.7 Zeitschrift für Bibliothekskultur / Journal for Library Culture • Digital Humanities und wissenschaftliche Bibliotheken

\title{
Gelehrte Forschungspraxis und digitale Bibliothek
}

Gerhard Lauer

Published on: Apr 20, 2021

License: Creative Commons Attribution 4.0 International License (CC-BY 4.0). 


\section{ABSTRACT}

Der Beitrag diskutiert vor dem Hintergrund der langen Geschichte und engen Verbindung von Gelehrsamkeit und Bibliothek, welche Folgen die digitale Transformation der Geisteswissenschaften für die Bibliotheken hat. Dafür folgt der Beitrag den Grundtechniken geisteswissenschaftlichen Arbeitens, dem Entdecken, Annotieren, Vergleichen, Referenzialisieren, Zusammenführen, Illustrieren und Darstellen. Leitende These ist, dass Bibliotheken mehr als bisher Teil des geisteswissenschaftlichen Forschungsprozesses werden müssen, jetzt aber eines grundlegend arbeitsteiligen Forschungsprozesses, in dem die Grenze zwischen Forschung und Service neu verhandelt werden muss.

The article discusses the consequences for libraries concerning the digital transformation of the humanities in light of the long history and close connection of scholarship with libraries. The article follows the basic methods and techniques of the humanities: discovery, annotation, comparison, referencing, amalgamation, illustration, and presentation. The guiding thesis is that libraries need to become more involved in the humanities' research process, and are indeed now becoming a fundamental part of the research process in which the boundaries between research and service need to be renegotiated.

Gelehrsamkeit braucht Bücher und Bücher brauchen Bibliotheken. Dieser uns so geläufige Zusammenhang hat eine keineswegs einfache (미‥ Hoepfner, 2002; König,_ Oikonomopoulou, \& Woolf, 2013), dafür lange Geschichte, die man schon etwa in den Etymologiae des Isidor von Sevilla aus dem frühen 7. Jahrhundert, dort im Sechsten Buch und Dritten Abschnitt, zu einem Gemeinplatz verdichtet finden kann. $\underline{1}$ Noch für die im 19. Jahrhundert entstehenden Geisteswissenschaften und ihre in direkter Konkurrenz zu den aufkommenden Naturwissenschaften entfalteten editorischen Grossforschungsprojekte ist die Verknüpfung der Gelehrsamkeit mit den Bibliotheken wesentlich. Gelehrsamkeit, Bibliotheken und Bücher gehören unverändert eng zusammen, auch wenn sich die tatsächliche Forschungspraxis schon länger von dieser Kurzformel entfernt hat.(Rebenich,2012). Gemeinplätze haben den kommunikativen Vorteil, Komplexität zu reduzieren, aber den intellektuellen Nachteil, etwa die Komplexität der Beziehung zwischen Gelehrsamkeit und Bibliothek zu reifizieren und das gerade dann, wenn die Traditionen der Gelehrsamkeit andere zu werden beginnen. Die digitale Transformation ist eine dieser grundlegenden Veränderungen der Gelehrsamkeit, die auch die Bibliotheken verändern werden. (Lauer,2019). Von dieser 
Veränderung ist im Folgenden zu handeln, genauer von den Veränderungen, die sich durch die veränderten Forschungspraxen in den Geisteswissenschaften ergeben. Vorauszuschicken ist dabei, dass diese neue Gelehrsamkeit erst ansatzweise zu einer Praxis verdichtet ist und je nach Disziplin unterschiedlich ausfällt. Die Archäologie und Linguistik haben schon länger andere, nicht nur historisch-hermeneutische Verfahren entwickelt und ihr Umgang mit Bibliotheken ist daher schon länger ein anderer als in den meisten geisteswissenschaftlichen Fächern.

Abstrakt lässt sich geisteswissenschaftliche Forschung in einzelne Elemente gelehrter Praxen zerlegen, Grundtechniken, die geisteswissenschaftliche Arbeit ausmachen. John Unsworth hat die Grundelemente, auch Primitive genannt, aus denen die gelehrte Praxis zusammengesetzt ist, wie folgt bezeichnet: Entdecken, Annotieren, Vergleichen, Referenzialisieren, Zusammenführen, Illustrieren und Darstellen. (Unsworth, 2000). Wie John Unsworth mit Recht schon im Jahr 2000 betont hatte, gelten diese elementaren Praxen auch unter den Bedingungen der Digitalität. Doch die Kontinuität der geisteswissenschaftlichen Arbeitsweisen gilt nur auf der vergleichsweise abstrakten Ebene, nicht auf der Ebene konkreter Forschungspraxis. Hier beginnt sich schon länger einiges zu ändern, so dass die Begriffe des Entdeckens, Annotierens, Vergleichens, Referenzialisierens, Zusammenführens, des Illustrierens und Darstellens eine andere Bedeutung annehmen. Es lohnt sich daher die Elemente der gelehrten Forschungspraxis im Einzelnen durchzugehen, um genauer zu ermitteln, was sich für die Bibliotheken ändern dürfte, ja vielfach schon geändert hat. Die leitende These ist, dass die Bibliotheken ihr alexandrinisches Zeitalter erst vor sich haben. Bibliotheken werden mehr als bisher Teil des geisteswissenschaftlichen Forschungsprozesses.

\section{Entdecken}

Die Gegenstände geisteswissenschaftlicher Forschung sind in einem Umfang und mit einer Geschwindigkeit zu entdecken, die sich selbst vor 20 Jahren kaum jemand vorzustellen vermochte, mag es sich um ein schwer zugängliches Mozart-Manuskript oder eine noch kaum entzifferte Keilschrifttafel handeln. $\underline{2}$ Die Retrodigitalisierung hat Kontinente der Kultur erschlossen und vielfach sind es Bibliotheken, die diese Quellen zur Verfügung stellen. Meta-Suchsysteme wie Europeana oder der WorldCat ermöglichen es, Übersicht über Quellen und Forschungsliteratur zu gewinnen, die bislang nur eingeschränkt oder gar nicht zugänglich waren. Das Suchen und Entdecken hat sich daher für fast alle geisteswissenschaftlichen Fächer verändert. Doch der Prozess des Suchens, Findens und Entdeckens war und ist bis heute ein eher privater Vorgang, der in den wissenschaftlichen Veröffentlichungen nur im Falle von 
spektakulären Funden thematisiert wird. Das ändert sich, wenn komplexere, computergestützte Suchverfahren wie reguläre Ausdrücke, Scraping-Verfahren oder Meta-Crawler verwendet werden. Für die Bibliotheken stellt sich dann die Frage, wie weit sie solche Suchen unterstützen und eine mögliche Dokumentation der jeweils gewählten Suchtechnik absichern könnten. Diese Notwendigkeit hat mit einer weiterreichenden Veränderung im geisteswissenschaftlichen Forschungsprozess zu tun, die man meist als Iteration bezeichnet. Gemeint ist, dass der Forschungsprozess im Sinne der Reproduzierbarkeit wiederholbar sein sollte, und dazu gehört dann unvermeidlich auch vielfach die Rekonstruierbarkeit, wie Forschungsgegenstände ermittelt wurden. Digitale Forschungsprozesse ermöglichen iterative Forschungsprozesse und binden auf diese Weise die Bibliotheken schon von Anfang an enger als bisher in den Forschungsprozess ein.

\section{Annotieren}

Die aufgefundenen Objekte der Forschung werden im Forschungsprozess vielfach angereichert, gerade auch im digitalen Prozess, sei es durch verbesserte Metadaten, durch detaillierte Auszeichnungen wie etwa XML oder auch TEI, sei es durch qualitative Annotationen inhaltlicher Aspekte. $\underline{3}$ Damit werden Objekte und Texte, wie sie in den Bibliotheken vorgehalten werden, verändert. Dies ist aber eine Veränderung im Verlauf der Forschung, die wiederum interessant für andere Forscherinnen oder Forscher ist. Im Sinne eines iterativen, prinzipiell wiederholbaren Forschungsprozesses wäre es daher wünschenswert, wenn Varianten eines Textes oder Objektes mit ihren jeweiligen Vorlagen zusammen angezeigt werden könnten. Dazu müsste es aber erst einmal einen Mechanismus für das Rück-Einspielen angereicherter Objekte und Texte in die bibliothekarischen Verwaltungssysteme geben und damit auch die Möglichkeit, Varianten als angereicherte Fassung aufzunehmen. Prüfverfahren wären zu etablieren, um das Bewahrenswerte zu identifizieren und dann aufzunehmen. Auch dies ist weit weniger ein technisches Problem denn eine Veränderung der Rollen der am Forschungsprozess beteiligten Instanzen, und damit auch eine Anfrage an die Funktion der Bibliotheken im Forschungsprozess.

\section{Vergleichen}

Dieses Rückspielen der angereicherten Objekte an die Bibliotheken hängt eng mit einem weiteren elementaren Forschungsschritt zusammen, dem Vergleichen. Wo Dinge zusammengeführt werden, dort können sie verglichen und in Gruppen eingeteilt werden. Derzeit erlauben Bibliotheken etwa die Abfrage von Sachgruppen, um Ähnlichkeiten in den Beständen aufzufinden. Aber hier ist mehr möglich, seit 
automatisierte Klassifikationsverfahren an Bedeutung gewinnen. Die Bibliotheken können schon heute verschiedene Klassifikationssysteme nutzen, um die Suchmöglichkeiten in ihren Katalogen grundlegend zu erweitern und komplexere Suchanfragen zu ermöglichen. Es gibt längst eine Reihe von Textmining- und Klassifikations- beziehungsweise Clusteringverfahren, um schon in den Katalogdaten Zusammengehörigkeitsmuster zu entdecken. Suchkataloge könnten also ihre Expertensuchmaske grundlegend um automatisierte Suchverfahren erweitern und ihre Bestände in Dashboards anzeigen. Auch damit würden Bibliotheken enger in den Forschungsprozess eingebunden werden, als dies in der etablierten geisteswissenschaftlichen Forschung gängig ist.

\section{Referenzialisierung}

$\mathrm{Zu}$ den Eigenheiten des iterativen Forschungsprozesses zählt auch eine stärkere Fokussierung auf Korpora. Nicht mehr Sammlungen, auch nicht das kanonische Einzelwerk, sondern repräsentative und balancierte Korpora liegen der neuen Forschung immer öfters zugrunde. Um die Übersicht zu behalten, müssen die Texte oder Objekte genauer als bisher referenzialisiert werden können, denn die Fundorte, Annotationen und vergleichend genutzten Forschungsgegenstände können auf sehr unterschiedlicher Ebene miteinander verglichen und dafür referenzialisiert werden, etwa wenn eine bestimmte Textpassage mit einem illustrierenden Kupferstich und dieser mit einem Objekt in einer universitären Sammlung in Verbindung gebracht wird. Verweise in Publikationen können schon jetzt mit eindeutigen Identifikatoren versehen oder Personen- und Ortsnamen disambiguiert werden. Daraus entstehen dann komplexe, maschinenlesbare Semantiken, die in verschiedenen Datenstandards wie RDF abgebildet und in Ontologien wie CIDOC-CRM systematisiert werden können. $\underline{4}$ Die zu Ontologien verdichteten Referenzialisierungen sind daher eine der Herausforderungen, denen sich auch Bibliotheken stellen müssen, wenn sie Teil dieser wachsenden Semantisierung der Referenzen werden wollen.

\section{Zusammenführen}

Dass stärker als bisher mit Korpora gearbeitet werden wird, bedeutet auch, dass in dem iterativen, auf Reproduzierbarkeit ausgelegten Forschungsprozess die entdeckten, gesammelten, angereicherten und referenzialisierten Texte und Objekte zusammenzuführen sind, auch über die Grenzen einzelner Bibliotheken hinweg. Die Texte und Objekte müssen für eine sinnvolle Korpusbildung nicht unbedingt aus ein und derselben Bibliothek stammen, sondern können aus verschiedenen Bibliotheken, Archiven oder Museen für die jeweilige Fragestellung zusammengetragen worden 
sein. Was analog nicht möglich ist, ist digital möglich, etwa die auf unterschiedliche Bibliotheken verteilte Manuskriptseiten einer mittelalterlichen Handschrift virtuell durch IIIF-Technologie so miteinander zu verknüpfen, dass für die Nutzenden das zusammenhängende Manuskript wieder am Bildschirm entsteht, das es einmal vor 1'000 Jahren war. Detaillierte Metadaten referenzialisieren dabei zurück auf die besitzenden Bibliotheken. Was für das einzelne Manuskript gilt, gilt auch für grössere Korpora. Denn die Nachnutzung durch andere ist dann nicht nur für Zwecke der Reproduzierbarkeit wichtig, sondern mehr noch für das Nutzen von Korpora durch die Forschungsgemeinschaft wie es etwa in der Korpuslinguistik länger schon Praxis ist. Wer mit diesen zusammengeführten Dokumenten oder Korpora weiterarbeiten möchte, braucht die Bibliotheken als unerlässliche Infrastruktur im Forschungsprozess. Sie sind der Ort, an dem die Zusammenführung stattfindet, vorgehalten und dokumentiert werden kann. Auch deshalb sind Bibliotheken Teil des Forschungsprozesses und nicht eine ihm vorgelagerte Infrastruktur.

\section{Illustrieren und Darstellen}

Die angereicherten, in Korpora zusammengeführten Texte und Objekte ermöglichen nicht nur den Aufsatz oder die Monographie als einzige Form der Darstellung von Forschungsergebnissen. Vielmehr kann der Forschungsprozess neben den Ergebnissen auch die Forschungswerkzeuge nachvollziehbar offenlegen. Korpora können in bibliothekarischen Repositorien für die weitere Forschung zur Verfügung gestellt, Werkzeuge auf Plattformen wie GitHub geteilt und mit den in Bibliotheken aufbewahrten Publikationen verknüpft, und Publikationen über Ontologiemodelle mit den Ressourcen und Forschungsprozessen verbunden werden. Mikroservices erzeugen Visualisierungen aus den aufgebauten Korpora, kurz, die Kette der Verknüpfungen und die Möglichkeiten der Visualisierung reichen weit über den in den Geisteswissenschaften üblichen Sammelband und die gedruckte Monographie hinaus. Wer, wenn nicht Bibliotheken sind dann der Ort, die geisteswissenschaftlichen Ergebnisse zugänglich zu halten. Und das braucht mehr als Bücherregale und Katalogsysteme.

Die Datafizierung auch des gelehrten Forschungsprozesses ist keine Zukunft, sondern in Abhängigkeit von den Institutionen, Instanzen und Akteuren mehr oder minder schon gegenwärtige Praxis, wenn auch in einem höchst unterschiedlichen Umfang. Ihr Gelingen hängt aber an einer Reihe von Richtungsentscheidungen, die auch die Bibliotheken betreffen, genauer in denen die Bibliotheken auch eine Stimme haben müssen, auch wenn die Positionen dazu derzeit noch kontrovers diskutiert werden. (z.B. Furger, 2016). Eine erste Entscheidung hängt an der Entwicklung und 
Durchsetzung von Standards, etwas, was Bibliotheken in ihrer Geschichte schon immer getan haben, damit die Bücher und Manuskripte wieder gefunden werden. Für den datenintensiven Forschungsprozess von heute bedeutet es, dass Datenstandards wie GND, TEI oder IIIF immer auch Standards sind, die mit darüber entscheiden, was Bibliotheken leisten können, um Forschungsprozesse zu unterstützen. Dass hier Bibliotheken mitsprechen sollten, scheint mir nahezuliegen.

Zweitens wird kaum etwas von den hier skizzierten Möglichkeiten Wirklichkeit werden, wenn für das Teilen von Daten keine am Gemeingut-Gedanken orientierten Regelungen entwickelt und gelebt werden. Die Konflikte um die Wissenschaftsschranken im Urheberrecht sind ein Hinweis darauf, wieviel hier auch bibliothekspolitisch zu tun bleibt, wenn Bibliotheken die neuen Forschungsprozesse unterstützen wollen. Die Forderung, dass Bibliotheken ihre Inhalte über die Grenzen einzelner Häuser hinweg vernetzen müssen, ist nicht neu. (Tochtermann, 2013). Doch noch sind viele der Ideen für eine wissenschaftliche Bibliothek der Zukunft mehr Wunsch als gelebte Wissenschaftspraxis.

Noch präziser und damit drittens geht es genau genommen nicht um Unterstützung durch die Bibliotheken, sondern um kollaborative Forschung mit den Bibliotheken. Während etwa Rechenzentren schon selbstverständlicher Teil des Forschungsprozesses in den Naturwissenschaften sind, sollten Bibliotheken Teil des gelehrten Forschungsprozesses werden, und das bis hin zur Veröffentlichung. Ihre Leistungen bestehen in sehr viel mehr als nur Bücher zu bewahren, auffindbar und zugänglich zu machen. Im Prozess der daten-intensiven Gelehrsamkeit sind sie bei fast jedem der elementaren Schritte beteiligt oder könnten an ihnen beteiligt sein. Dass das auch ein Umdenken in den philosophischen Fakultäten impliziert, sei hier hinzugefügt.

Wir leben in einem alexandrinischen Zeitalter, das eine nie gekannte Fülle an Gelehrsamkeit, Büchern und Bibliotheken verknüpfen kann. Wie stark und wie zügig die hier skizzierten Änderungen im gelehrten Forschungsprozess Wirklichkeit werden, ist schwer abzuschätzen. Die Persistenz werkästhetischer Forschungsunternehmen in zumeist von nationalen Kontexten abhängigen Forschungsprozessen, die Kultivierung des angeblich auf Humboldt zurückgehenden Gelehrtenideals der Arbeit in „Einsamkeit und Freiheit“ und das bibliothekarische Selbstverständnis als die buchzentrierte Infrastruktur lässt viele der inspirierenden Möglichkeiten innovativer Forschung mit und durch Bibliotheken nur schwer vorankommen. (ㅇomers, 2017). Wen das beruhigt, der sieht nicht, was die grossen Wissenschaftsverlage längst erkannt 
haben, dass nämlich sie die Forschungsinfrastruktur der Zukunft auch für die Geisteswissenschaften sein wollen und mit aller Aggressivität den Bibliotheken ihre Rolle streitig machen werden, ja schon machen. Wenn wir wollen, dass Wissenschaft ein Gemeingut, Forschung ein offener Prozess und Ergebnisse geteilte Erkenntnisse bleiben, dann müssen Bibliotheken die Aufgabe, die Forschungsinfrastruktur der gelehrten Fächer zu werden, annehmen.

\section{Footnotes}

1. http://www.monumenta.ch/latein/text.php?

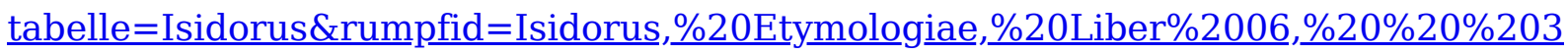
$\underline{\text { \&level }=4 \text { \&domain }=\text { \&lang }=0 \text { \&links }=\text { \&inframe }=1 \text { \&links }=1 \text { \&hide apparatus }=1}$

2. Vgl. die Digitale Mozart-Edition, https://mozarteum.at/digitale-mozartedition/\#info oder die Cuneiform Digital Library Initiative, https://cdli.ucla.edu/.

3. Vgl. Extensible Markup Language, https://www.w3.org/TR/xml11/ oder die Text

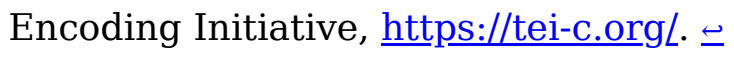

4. Vgl. das RDF-Schema, https://www.w3.org/TR/rdf-schema/, oder das Referenzmodell für Kulturelles Erbe, http://www.cidoc-crm.org $L . \pm$

\section{Citations}

1. Hoepfner, W. (2002). Antike Bibliotheken. Mainz: von Zabern. $\subseteq$

2. König, J., Oikonomopoulou, K., \& Woolf, G. (2013). Ancient libraries. Cambridge University Press. $\bullet$

3. Rebenich, S. (2012). Theodor Mommsen und Adolf Harnack. Berlin, Boston: De Gruyter. https://doi.org/10.1515/9783110812558

4. Lauer, G. (2019). Ist da eine Veränderung in den Geisteswissenschaften? : Infrastrukturen und ihre Folgen für die Praxis der Geisteswissenschaften. In M. Huber, S. Krämer, \& C. Pias (Eds.), Forschungsinfrastrukturen in den digitalen Geisteswissenschaften: Wie verändern digitale Infrastrukturen die Praxis der Geisteswissenschaften? (pp. 27-38). Frankfurt a. M.: Universitätsbibliothek Johann Christian Senckenberg. Retrieved from urn:nbn:de:hebis:30:3-519014 
5. Unsworth, J. (2000). Scholarly Primitives: what methods do humanities researchers have in common, and how might our tools reflect this? Retrieved from https://johnunsworth.name/Kings.5-00/primitives.html $\subseteq$

6. Furger, M. (2016). Bibliotheken: Weg damit! Das Internet mache Bibliotheken überflüssig, sagt der Chef der ETH-Bibliothek im Interview. Entweder sie räumen ihre Bücherbestände aus und erfinden sich neu - oder sie werden verschwinden. NZZ Am Sonntag Vom 7.2. Retrieved from https://nzzas.nzz.ch/hintergrund/bibliotheken-und-buecher-weg-damit-meint-rafaelball-ld.147683?reduced $=$ true $\subseteq$

7. Tochtermann, K. (2013). Eine neue Sicht auf die Bibliothek der Zukunft/Zehn Thesen zum zukünftigen Profil von wissenschaftlichen Informationsinfrastruktureinrichtungen mit überregionaler Bedeutung. BuB Forum Bibliothek Und Information, (11-12). Retrieved from https://b-u-b.de/neue-sicht-aufwissenschaftliche-bibliothek-zukunft-zehn-thesen/ $\triangleq$

8. Somers, J. (2017). Torching the Modern-Day Library of Alexandria. The Atlantic, April 20. Retrieved from https://www.theatlantic.com/technology/archive/2017/04/thetragedy-of-google-books/523320/ $\boxminus$ 